\title{
LE FAIT DIVERS: UNE NARRATION DÉFAILLANTE?
}

\section{LE FAIT DIVERS: UN GENRE, UNE RUBRIQUE, UN TYPE DE TEXTE OU UN CONCEPT?}

Le fait divers: un genre très ancien, ${ }^{1}$ mais toujours aussi difficile à décrire et à définir. Histoires relatant des accidents, meurtres, viols que tout le monde lit mais que personne n'avoue lire... Même si " cette rubrique est encore dévalorisée socialement » (Lits 2001 : 1) et malgré le fait que les faits divers " occupent traditionnellement la place la moins noble dans la hiérarchie de l'information » (Bégorre Bret et al. 2004 : 1), la quantité des articles appartenant à cette rubrique augmente et ils sont passés dans d'autres médias, p. ex., à la télévision, où ils sont « désormais souvent abordés en première partie de journal télévisé » (ibid : 2).

Le fait divers « raconte une histoire qui se détache sur le fond de toutes celles qui ne sont pas racontées et qui sont la norme » (ibid : 4), donc une histoire hors du commun. Bégorre Bret et al. citent Barthes selon qui « le fait divers (...) procéderait d'un classement de l'inclassable, il serait le rebut inorganisé des nouvelles informes; son essence serait privative, il ne commencerait d'exister que là où le monde cesserait d'être nommé, soumis à un catalogue connu (politique, économie, guerre, spectacles, science, etc.) » (ibid : 10).

Lits (2001) et Adam (2001) constatent qu'il s'agit d'un genre peu étudié par les chercheurs qui traitent de corpus souvent trop limités. Selon Lits, qui décrit son expérience avec des étudiants originaires d'autres pays, il s'agit d'une catégorie fortement marquée culturellement; il se demande s'il ne s'agit pas d'un genre strictement francophone. Ces étudiants originaires surtout d'Espagne, du Portugal, de l'Afrique francophone et des pays latino-américains, après avoir appris que l'objet du séminaire d'analyse de presse porterait sur le fait divers, n'ont pas compris quel serait l'objet de l'étude, «non en raison de leur incompréhension du français, mais par méconnaissance du terme générique qui n'était pas transposable comme tel dans leur réalité culturelle» (2001:2). Dans les journaux de leurs pays respectifs, les faits divers sont classés différemment et ne sont pas rubriqués. Selon Lits, «l'échantillon d'étudiants non francophones ne dispose donc pas de la compétence générique pour comprendre l'objet de l'analyse qui leur est proposé, puisque dans leur culture et dans leur langue, cette catégorie journalistique n'existe pas » (ibid : 3).

Pourtant, López Alonso et Séré décrivent une expérimentation faite auprès d'apprenants espagnols: « Pour faciliter la reconnaissance du type, les textes ont été

\footnotetext{
* Adresse de l'auteur : Filozofska fakulteta, Oddelek za romanske jezike in književnosti, Aškerčeva 2, 1000 Ljubljana, Slovénie. Mél: meta.lah@guest.arnes.si

${ }^{1}$ Dans la presse francophone, les premiers faits divers apparaissent à la fin des années 1830 (Dubied $2001: 2-3$ ).
} 
présentés sous leur forme authentique, pour le fait divers et la recette de cuisine il n'y a eu aucune difficulté à identifier le prototype textuel» (1996:444).

Selon nos expériences, les apprenants slovènes n'ont aucune difficulté à reconnaître le genre. En Slovénie (comme dans certains pays, mentionnés par Lits, p. ex. l'Italie, l'Espagne ou certains pays d'Amérique du Sud), ce genre de texte apparaît dans la rubrique kronika (la chronique) ou črna kronika (la chronique noire). Il faut donc annoncer aux apprenants un "članek iz črne kronike » (un article de la chronique noire) et ils reconnaissent le type de texte. Nous supposons que même si dans différentes langues les faits divers sont nommés et rubriqués différemment, le concept reste le même. Serait-il donc possible de trouver dans différentes cultures et différentes langues des faits divers comparables?

\section{LE FAIT DIVERS: UN TEXTE DE TYPE NARRATIF OU UN MÉLANGE DE PLUSIEURS TYPES?}

En général, on aurait tendance à dire que le fait divers est un texte de type narratif. Ce type de texte, comme le disent Bégorre Bret (2004:4) et al., raconte une histoire qui se détache des autres. Dans ces textes "l'irruption de l'inhabituel dans le quotidien fait naître l'intuition d'une causalité à l'oeuvre » (ibid.). Chaque fait divers contient donc cette composante d'inhabituel dont parle Eco, que nous citerons un peu plus loin.

Dans les faits divers, les auteurs utilisent un lexique de caractérisation des personnages, des lieux et des moments et les indicateurs temporels, ce qui est typique pour les textes narratifs (Tagliante 1994 : 131). En principe, un fait divers contient tous les " ingrédients narratifs, prescrits par l'ancienne rhétorique (qui, quand, où, comment, à qui, pourquoi)» (Petitjean 1987 : 84).

Pour pouvoir analyser les textes regardons d'abord la définition d'une séquence narrative:

\begin{tabular}{|c|c|c|c|c|}
\hline \multicolumn{5}{|c|}{$\begin{array}{c}\text { Récit } \\
\text { Séquence } \\
\text { narrative }\end{array}$} \\
\hline $\begin{array}{c}\text { Situation } \\
\text { initiale } \\
\text { (Orientation) }\end{array}$ & $\begin{array}{c}\text { Complication } \\
\text { Déclencheur } 1 \\
\text { (noeud) }\end{array}$ & $\begin{array}{l}\text { Action ou } \\
\text { Evaluation }\end{array}$ & $\begin{array}{l}\text { Résolution } \\
\text { Déclencheur } 2 \\
\text { (dénouement) }\end{array}$ & Situation finale \\
\hline $\mathrm{Pn}_{1}$ & $\mathrm{Pn}_{2}$ & $\mathrm{Pn}_{3}$ & $\mathrm{Pn}_{4}$ & $\mathrm{Pn}_{5}$ \\
\hline
\end{tabular}

(Adam $1994:$ 104)

Tisset (2000 : 181) résume les caractéristiques de la séquence narrative de la façon suivante: 
«- Mise en intrigue et unité d'action. Un assemblage d'événements et d'actions ayant un commencement, un milieu et une fin; tout est élaboré en vue du dénouement.

- Temporalisation et causalité. Les événements se succèdent de telle sorte qu'ils semblent déterminés les uns par rapport aux autres; les événements postérieurs trouvent leur causalité dans les événements antérieurs.

- Unicité d'un sujet et transformation des prédicats: les prédicats d'être, d'avoir ou de faire avec réussite ou échec.

Pour former un récit, les séquences peuvent être coordonnées linéairement, enchâssées ou montées en parallèle. »

Eco reprend la définition de Van Dijk et souligne la composante d'inattendu, inusuel ou étrange: « ...une narration est une description d'actions qui requiert pour chaque action décrite un agent, une intention de l'agent, un état ou un monde possible, un changement avec sa cause et le propos qui le détermine; on pourrait ajouter à cela des états mentaux, des émotions, des circonstances; mais la description est importante [...] si les actions décrites sont difficiles et seulement si l'agent n'a pas un choix évident quant au cours des actions à entreprendre pour changer l'état qui ne correspond pas à ses propres désirs; les événements qui suivent cette décision doivent être inattendus, et certains d'entre eux doivent apparaitre inusuels ou étranges » (1985 : 137).

Dans le Dictionnaire d'analyse du discours, Adam, qui est l'auteur de l'article, choisit comme exemple de séquence narrative une " brève »: " Ainsi, dans cette brève journalistique de F. Fénélon: 'A peine humée sa prise (1), A. Chervel éternua (2) et, tombant du char à foin (3) qu'il ramenait de Pervencheres (Orne), (4) expira (5).' La proposition (1) apparaît comme le noeud $\left(\mathrm{Pn}_{2}\right)$ d'un récit qui commence sans exposé de sa situation initiale: c'est parce qu'il prend du tabac à priser (cause volontairement choisie) que le malheureux Chevrel éternue (conséquence involontaire). La proposition (2) apparaît comme la réaction $\mathrm{Pn}_{3}$. La proposition (4), insérée tardivement dane le cours de la phrase (la parenthèse indicatrice du lieu se trouve généralement plutôt en tête), explique ce que fait le personnage sur le char; soit la situation initiale du récit $\left(\operatorname{Pn}_{1}\right)$. Le lien entre le participe présent de (3) et le passé simple final (5) est un lien de cause à effet dans lequel (3) apparaît comme le dénouement $\mathrm{Pn}_{4}$ et (5) comme la situation finale $\mathrm{Pn}_{5}$. »

Si le fait divers raconte une histoire (qui répond à toutes les questions posées habituellement et, en plus, mentionne quelque chose d'inhabituel), on pourrait supposer que le fait divers est une narration. Pourtant, Petitjean souligne que le fait divers est hétérogène, du point de vue énonciatif (il rapporte une pluralité des voix) et du point de vue textuel, parce qu'il est " dominé par une structure narrative qui implique actions, descriptions, dialogues et commentaires et [...] n'est pas dépourvu d'enjeux explicatifs et argumentatifs. » (Petitjean 1987: 73-74). Dans cet article, c'est surtout le côté textuel qui nous intéresse: nous allons nous concentrer sur l'analyse des faits divers dans différentes langues et essayer de voir quelle y est la place de la narration. 


\section{ANALYSE D'UN FAIT DIVERS DANS DIFFÉRENTES LANGUES}

\subsection{Présentation du problème}

Nous partons de l'hypothèse que le fait divers, portant sur un même thème, a une structure textuelle comparable dans différentes langues et contient au moins une partie (une séquence) narrative. Nous avons rassemblé un corpus de faits divers portant sur le même sujet dans les langues suivantes: français, espagnol, italien, anglais, allemand, slovène et croate. ${ }^{2}$

Le choix du fait divers a été difficile. En lisant la presse internationale tout l'été 2008, nous avons trouvé deux faits divers " internationaux »: le premier portant sur le crash d'avion à Madrid et le deuxième qu'on pourrait classifier comme "insolite ", à savoir l'histoire de l'effigie d'Hitler dans le musée Tussaud à Berlin, décapitée tout de suite après son exposition. Ce fait divers se prête à l'analyse: il a été publié dans les médias du monde entier, de plus, il ne s'agit pas d'un fait divers sanglant: il n'y a pas de vraie victime puisque la « victime » est une statue de cire.

\subsection{Analyse des faits divers}

En lisant les différents articles, il est possible de constater qu'en général les faits sont rapportés de deux manières; "selon qu'ils se présentent sous une forme condensée ou expansée. Les premiers, non signés, [...] se contentent de reproduire, sans trop de transformations, le récit de l'agence. Textes brefs, ils ont la forme d'un résumé expurgé au maximum d'expansions narratives, descriptives, commentatives et réduisent le dialogue à des citations entre guillemets [...]. Les seconds sont généralement signés et ont la forme, entre le résumé d'ouverture et la séquence de clôture, d'une expansion narrative analeptique racontée par une voix ou par plusieurs. » (Petitjean 1987 : 83).

Nous avons retenu deux exemples d'articles plus courts. Le premier texte est un des rares exemples d'articles ne contenant qu'une séquence narrative:

\section{Exemple 1: Hitler Without a Head ${ }^{3}$}

(1) In Berlin, in a newly opened section of a museum, (2) a 41 year old man cut off Adolf Hitler's head. (3) The Hitler figure was modeled from wax. (4) The case happened shortly after the exhibition was opened to the public.

(5) The police were alarmed (5') and arrested the offender. (6) The patrons of the exhibition insist that the Hitler figure has to be here and it should not be ignored.

\footnotetext{
${ }^{2}$ Il est intéressant de voir comment les faits divers choisis sont rubriqués: "shortnews", "flashactu", "article", "story", "dnevne vijesti" (les nouvelles du jour), "članek" (article), "panorama" et même "gente y TV". Les articles n'apparaissent pas dans la rubrique "faits divers". Ceci confirme que les faits divers sont "le plus souvent nationaux" (Bégorre Bret et al. 2004 : 2).

${ }^{3}$ Ce texte provient d'un journal en ligne allemand. Nous l'avons retenu à cause de sa structure: à part la dernière phrase, qui est explicative, on n'y trouve que de la narration. Pour rendre le texte plus clair, nous avons adapté la proposition (5); dans le texte original: "The police arrested the offender after they alarmed them."
} 
L'histoire est simple: après beaucoup de polémiques, les responsables du musée Tussaud de Berlin ont décidé d'exposer la statue d'Hitler. En signe de protestation, le deuxième visiteur a décapité la statue. Il a été par la suite arrêté par la police.

La chronologie des faits est la suivante:

(1) la direction du musée décide d'exposer la statue de cire

(2) le musée ouvre ses portes

(3) le visiteur décapite la statue

(4) la police est alertée

(5) la police arrête le « criminel».

Selon cette chronologie la proposition (3) serait le point culminant de l'histoire, $\mathrm{Pn}_{3}$ (action). Même si chronologiquement la proposition (2) vient après (1), c'est la proposition (1) qui, à notre avis, représenterait le noeud - $\mathrm{Pn}_{2}$ (lien de cause à effet - c'est à cause de cette décision que le visiteur agit de la sorte) et la proposition (2) la situation initiale $\mathrm{Pn}_{1}$. La proposition (4) - dénouement, $\mathrm{Pn}_{4}$, nous mènerait à la situation finale, la proposition (5), $\mathrm{Pn}_{5}$, le coupable est arrêté.

Dans l'exemple 1, l'auteur commence par $\mathrm{Pn}_{1}$ : (le musée ouvre ses portes), continue avec $\mathrm{Pn}_{3}$. La proposition (3) répète et complète l'information qui correspond à $\mathrm{Pn}_{1}$. La proposition (5) représente $\mathrm{Pn}_{4}$ et la proposition (5') $\mathrm{Pn}_{5}$. Dans la proposition (6), l'auteur ajoute une autre information: la cause de ce qui s'est produit - cette proposition n'entre pas dans le schéma de la séquence narrative proprement dite.

Exemple 2: Berlin : la statue d'Hitler sera réparée ${ }^{4}$

/1/ Trois minutes après l'ouverture du musée, en plein coeur de la capitale allemande, un Berlinois avait bousculé deux membres du personnel et arraché la tête de la figure du dictateur nazi en criant « plus jamais la guerre! ».

/2/ Pour expliquer son acte, cet ancien policier, âgé de 41 ans et aujourd'hui au chômage, raconte dans plusieurs journaux avoir fait un pari avec ses copains et avoir « eu la trouille » avant de s'attaquer à la statue.

La séquence narrative n'est pas complète, elle ne contient que la première partie de l'histoire (partie /1/). L'auteur ajoute plusieurs informations: temps et lieu exact du fait (trois minutes après l'ouverture, en plein coeur de la capitale) - ces informations complètent Pn1, la description du fait (bouscule deux membres..., en criant...) - ce qui complète Pn3. Il n'y a pas d'informations sur les actions de la police. L'auteur termine par une séquence explicative ${ }^{5}$ (partie $/ 2 /$ ):

Pourquoi le "criminel" a-t-il commis cet acte? - Parce qu'il a fait un pari...

\footnotetext{
${ }^{4}$ Nous avons omis les chapeaux, les surtitres et les sous-titres des articles; on y trouve dans la plupart des cas les mêmes informations que dans les articles.

${ }^{5}$ Le schéma de la séquence explicative a, selon Adam (1997 : 132) quatre parties: schématisation initiale, problème (question): pourquoi $\mathrm{X}$ ? comment $\mathrm{X}$ ?, explication (réponse): parce que..., conclusion.
} 
L'exemple 3 contient quatre parties:

Decapitata statua di Hitler museo delle cere di Berlino

/1/ (1)Salta la testa di Hitler al museo delle cere di Berlino. (2) A pochi minuti dall'inaugurazione del Madame Tussaud della capitale tedesca, (3) un uomo ha decapitato la statua del Führer. (4) Ad agire, un visitatore di 41 anni di Berlino, arrestato subito dopo dalla polizia. (5) L'uomo avrebbe agito in segno di protesta.

/2/ L'arrivo della statua di Adolf Hitler al museo delle cere ha suscitato forti polemiche. Molti, infatti, ritengono offensivo esporre la rappresentazione del dittatore in pubblico.

/3/ La statua, prima di essere deturpata, ritraeva Hitler negli ultimi giorni della sua esistenza, seduto alla scrivania, nel bunker dove si tolse la vita nel 1945. /4/ Il nuovo museo delle cere di Berlino, aperto vicino alla Porta di Brandeburgo, è la terza succursale del Madame Tussaud in Europa, dopo Londra e Amsterdam. Nel mondo, in tutto sono otto.

La partie /1/ est la partie narrative: la première proposition (1) sert d'introduction et reformule le titre. La proposition (2) représente $\mathrm{Pn}_{1}$ (situation initiale), la proposition (3) l'action, $\mathrm{Pn}_{3}$ et la proposition (4) la situation finale $\mathrm{Pn}_{5}$. "Un visitatore di 41 anni di Berlino" complète la description de "l'uomo" de la proposition (3). La proposition (5) explique le comportement de l'homme et sert donc d'explication (pourquoi l'homme a-t-il agi de la sorte?).

Dans la partie /2/, l'auteur décrit les polémiques avant l'exposition de la statue d'Hitler, ce qui pourrait représenter le noeud de la narration.

Les parties /3/ et /4/ sont des parties descriptives. Dans /3/, il s'agit de la description de la statue et dans /4/ de la description du musée. La dernière partie a une visée informative.

Exemple 4: Wax Hitler Loses Its Head

/1/ (1) A wax model of Adolf Hitler at a new Madame Tussauds in Germany was decapitated (2) by a Berlin man (3) soon after the museum opened today, AFP reports. (4) The man, (5) who was arrested, (4') "wanted to protest against Hitler's figure being on show," police said. /2/ The model had sparked controversy, but the director of the German history exhibit said it wouldn't make sense to leave the dictator out, reports the BBC.

"Seeing as we are portraying the history of Germany, we could hardly have left him out," said a museum spokesman. Added a rep for a German Jewish council, "Erasing him from history is not going to bring the perished ones back." /3/ The model was perched behind a desk so people wouldn't pose with it or vandalize it. It portrayed Hitler in defeat, soon before his suicide in his bunker.

L'exemple 4 est composé de trois parties: dans la narration (partie /1/): les propositions (1) et (2) représentent l'action, la proposition (5) représente la situation finale. La proposition (4) est composée de deux parties et, comme dans les faits divers 
précédents, explique le comportement du Berlinois. La partie /2/ explique la décision du musée (explication) et la partie /3/ est descriptive: il s'agit d'abord de la description de la position de la statue et ensuite de la description de la statue elle-même.

Ce fait divers est très intéressant du point de vue énonciatif. Il s'agit d'un fait divers polyphonique, où l'événement est raconté par plusieurs voix (Petitjean 1987 : 74). La narration est faite par les para-énonciateurs (ibid.): d'une part la police (« wanted to protest against Hitler's figure... ») et, d'autre part, les représentants du musée et de la communauté juive (" seeing as we are portraying... », " Erasing him from history... »). Selon Petitjean, l'intérêt de cette polyphonie énonciative est double, " elle sert à créer un effet de vérité en donnant au lecteur l'impression d'une complétude informative» (ibid. : 76).

\section{Exemple 5: Muzej Madame Tussaud: Posjetitelj Hitleru otkinuo glavu}

/1/ (1) Nakon nedavnog otvorenja galerije Madame Tussaud u Berlinu (2) dogodio se neobičan incident. (2') Naime, jedan 41-godišnji posjetitelj je otkinuo glavu najpoznatijem izlošku, figuri Adolfa Hitlera.

(3) Glasnogovornik berlinske policije je izjavio kako je počinitelj_odmah uhićen.

/2/ Posjetitelji su nakon višemjesečnih kontroverzi, u četvrtak, prvi put u galeriji imali prilike vidjeti bivšeg njemačkog kancelara i vođe tzv. Trećeg Reicha Adolfa Hitlera.

/3/ Hitler je prikazan kao uništen starac na kraju života.Osim toga, on je jedina figura uz koju nije dopušteno poziranje, a i fotografiranje je strogo zabranjeno « iz pijeteta zbog milijuna žrtava nacionalsocijalističkog režima », kako stoji na ploči u prostoriji u kojoj se nalazi Hitlerova figura. /4/ Uz Hitlera, koji je smješten u odvojenoj prostoriji koja je projektirana prema Hitlerovom posljednjem utočištu u podzemnom bunkeru kancelarskog ureda, nova podružnica galerije voštanih figura prikazuje još sedamdesetak osoba iz njemačkog i svjetskog javnog života poput Angele Merkel ili Siegmunda Freuda. /5/ Uprava berlinske podružnice londonske galerije voštanih figura na vrijeme je reagirala na upozorenje nekih dijelova javnosti te je uvela posebne mjere koje se tiču isključivo Hitlerove figure.

L'article, rédigé en langue croate, est composé de cinq parties:

- /1/ la partie narrative,

- $\quad / 2 /$ le journaliste explique qu'après plusieurs mois de polémiques sur le sujet les visiteurs ont pu voir la statue d'Hitler (explication),

- /3/ la description de la statue d'Hitler (il est représenté à la fin de sa vie, comme un homme proche de la mort) et l'explication sur l'interdiction de prendre des photos - /4/ l'énumération des autres personnes ayant leur statue dans le musée (description),

- /5/ l'explication sur les mesures de sécurité quant à la statue d'Hitler.

L'article est intéressant du point de vue textuel. La partie narrative est relativement pure du point de vue narratif: les propositions (1) et (2) représentent la situation 
initiale et l'action (en mentionnant, dans la proposition (2), un "incident" inhabituel). La proposition (2') complète l'action en donnant des informations supplémentaires sur le lieu et l'auteur des faits. La proposition (3) est la situation finale: selon le porte-parole de la police, l'homme a été arrêté.

Dans les parties /2/ - /5/ il est plus difficile de reconnaitre le type textuel: il y a un mélange d'explication et de description. Le but de l'auteur est sans doute d'informer.

Exemple 6: Voščenemu Hitlerju odtrgal glavo

/1/ Berlin - (1) Le nekaj ur po slavnostnem odprtju razstave muzeja Madame Tussaud, (1') ki vključuje tudi voščeno lutko Adolfa Hitlerja, (2) je 41-letni_obiskovalec voščeni lutki odtrgal glavo. (3) $\mathrm{S}$ tem je želel protestirati proti umestitvi spornega zgodovinskega lika v razstavo. (4) 41-letnika, ki je najprej odrinil varnostnika, da se je prebil do lutke in ji odtrgal glavo, (5) so policisti priprli.

/2/ Vključitev lutke Adolfa Hitlerja v razstavo je vzbudila številne kritike in negodovanja v državi, kjer so nacistični simboli prepovedani. Organizatorji so se branili z argumentom, da bi bilo težko prikazati nemško zgodovino brez vključitve Hitlerjevega lika. Lutka, ki je zastražena, kaže Hitlerja, ko sedi za mizo v svojem berlinskem bunkerju. Hitler sedi za mizo iz še enega razloga - da se obiskovalci ne bi mogli slikati z lutko.

/3/ Natalie Ruoss iz muzeja Madame Tussaud je dejala, da so pred končno odločitvijo, ali vključiti Hitlerjev lik v razstavo ali ne naredili raziskavo in tako Berlinčane kot tudi turiste spraševali za mnenje. Iz odgovorov je razvidno, da je Hitler ena izmed oseb, ki si jih Berlinčani želijo videti na razstavi, je končno odločitev muzeja komentirala Roussova.

Comme les faits divers analysés ci-dessus, cet article, rédigé en slovène, commence par une séquence narrative. La proposition (1) - situation initiale ( peu après l'ouverture du musée), est complétée par l'information que dans ce musée on peut voir la statue d'Hitler (1') et continue avec la proposition (2) - action (un visiteur de 41 ans décapite la statue). La proposition (3) a une visée explicative (il a commis cet acte en signe de protestation...). La proposition (4) contient une information supplémentaire quant aux faits divers précédents (le visiteur a bousculé le gardien). La proposition (5) représente la situation finale (le coupable est maîtrisé et placé en garde à vue).

Dans la partie /2/ le journaliste présente les critiques en relation avec l'exposition de la statue du dictateur et les arguments des organisateurs. La partie /3/ complète la partie /2/ avec les propos de Natalie Ruoss du musée Tussaud qui explique pourquoi les organisateurs ont décidé de présenter la statue. Les deux parties donnent la réponse à la question "Pourquoi le musée a-t-il exposé la statue?" et ont donc une visée explicative.

Exemple 7: Museumsbesucher reisst Hitler den Kopf ab

/1/ (1) Der 41-Jährige hatte das Museum nahe dem Brandenburger Tor gegen $10 \mathrm{Uhr}$ betreten und die Figur angefasst. (1') Als ein weiterer Besucher ihn davon abhalten wollte, 
kam es zu einem Handgemenge. (1") Schliesslich riss der Mann der Wachsfigur den Kopf ab. (2) Er war der zweite Gast überhaupt, der nach der Eröffnung um zehn Uhr in das Museum gekommen war, (3) wie ein Museumsmitarbeiter berichtet.

(4) Die Polizei konnte den in Berlin wohnenden Mann im Museum festnehmen. (5) Nach ersten Erkenntnissen wollte er gegen die Ausstellung demonstrieren.

Kontroverse um Ausstellungsobjekt

/2/ Nach einer Pressevorbesichtigung am Mittwoch hatte es eine Kontroverse über die Frage gegeben, ob der Diktator überhaupt präsentiert werden dürfe. Die Kritiker werfen der Museumsleitung Effekthascherei vor. Die Figur sitzt in einem Diorama, das den Führerbunker im Jahr 1944 darstellt. Die Museumsverwaltung hatte erklärt, das Szenario dürfe weder berührt noch fotografiert werden.

/3/ Das neue Berliner Wachsfigurenkabinett ist nach London und Amsterdam die dritte Tussaud-Niederlassung in Europa und die achte weltweit.

La partie narrative introduit le fait divers: les phrases (1), (1') et (1') couvrent l'action: un visiteur de 41 ans est entré dans le musée à 10 heures environ et a décapité la statue, malgré l'intervention d'un autre visiteur avec lequel ils en sont venus aux mains. La phrase (2), surtout la deuxième proposition « der nach der Eröffnung... » correspond au noeud: le musée ouvre ses portes. La proposition (4) décrit la situation finale: la police a interpellé l'homme. La phrase (5) a une visée explicative et donne les raisons quant au comportement de l'interpellé.

La partie /2/ présente les polémiques et les critiques, mentionnées dans les faits divers précédents et décrit la façon dont Hitler est présenté.

La partie /3/ donne des informations sur le nombre de musées en Europe et dans le monde.

Exemple 8: Hitler, decapitado

/1/ (1) Un hombre de 41 años ha sido detenido (2) por arrancar la cabeza a la figura de cera de Adolf Hitler (3) en el Museo Tussaud de Berlín , (4) durante el primer día en que ha estado abierto al público, (5) ha informado hoy la policía.

/2/ La presencia de la figura de Hitler en el Museo Tussaud había generado duras críticas. Los responsables del museo se han defendido diciendo que Hitler pertenece a la historia alemana y que, además, la figura lo muestra ya en su época de decadencia, encerrado en su bunker y rodeado por el estruendo de bombas aliadas, y que no se presta para que pueda crearse un culto por parte de ultraderechistas. Pese a esas explicaciones, muchos políticos locales habían calificado de mal gusto la presencia de la figura de Hitler en la exposición. La figura de Hitler no puede ser tocada ni fotografiada, según las reglas impuestas por los responsables del museo.

/3/ Según la policía, el responsable de la decapitación vive en el barrio de Kreuzberg, un lugar emblemático por su multiculturalidad y porque sus habitantes suelen votar a la izquierda. El hombre al parecer quería manifestarse contra el hecho de que la figura de Hitler formara parte de la exposición del museo. /4/ (1) El detenido se acercó sobre las 
10 de la mañana a la figura de Hitler (2) y cuando se dispuso a tocarla (3) otro visitante trató de disuadirlo, (4) por lo que se produjo una escaramuza entre ambos. (5) Finalmente, el hombre de Kreuzberg le arrancó la cabeza a Hitler.

/5/ La filial del Museo Tussaud en Berlín es la tercera en Europa, después de la de Londres y Amsterdam, y la octava en el mundo.

L'exemple 8 contient deux séquences narratives: l'une au début /1/ et l'autre vers la fin de l'article /4/. La première commence par la situation finale (1) (l'homme est arrêté), continue par l'action (2) (pour avoir décapité la statue). Les propositions (3) et (4) complètent la proposition (2) en donnant la réponse aux questions où? et quand? et la proposition (5) explique d'où vient l'information.

La partie /4/ apporte des informations supplémentaires à la partie /1/. Nous y trouvons des informations que nous n'avons pas trouvées dans les faits divers analysés précédemment: l'action y est décrite de manière plus détaillée: il y a une information relative au temps de l'incident et à un autre visiteur qui a essayé - sans succès, d'ailleurs - d'empêcher l'homme d'agir.

La partie /2/ apporte des informations sur les critiques formulées lors de l'exposition de la statue et la justification du musée. Dans la partie /3/, il y a la description du milieu dans lequel vit le coupable et les raisons de son comportement.

La dernière partie /5/ contient des informations sur les filiales du musée.

\subsection{Analyse des faits divers- résumé}

Nous avons analysé huit fait divers: deux textes brefs, ayant la forme d'un résumé (exemples 1 et 2 ) et six faits divers plus longs (exemples de 3 à 8 ). La structure narrative prédomine dans les exemples 1 et 2 : dans l'exemple 1 il y a une seule proposition explicative (la dernière phrase: "The patrons... »). L'exemple 2 commence par la séquence narrative et continue par une séquence explicative.

Dans les faits divers plus longs, il est possible de distinguer au moins une séquence narrative, toujours au début de l'article. L'exemple 8, Hitler decapitado, contient deux séquences narratives, la première au début et la deuxième vers la fin de l'article: l'auteur y répète l'histoire du début, en donnant plus de détails sur le sujet.

Les séquences narratives ne contiennent pas toujours tous les éléments de l'exemple 1 que nous avons pris pour modèle.

Le contenu des autres séquences est pratiquement identique dans la plupart des faits divers mentionnés. Les thèmes évoqués sont:

- les raisons du comportement de l'homme: "pour expliquer son acte... » (ex. 2), « l'uomo avrebbe agito in segno di protesta » (ex. 3), « the man wanted to protest... » (ex. 4), «s tem je želel protestirati... » (ex. 6), « Nach ersten Erkentnissen wollte er [...] demonstrieren » (ex. 7), « El hombre al parecer quería manifestarse contra el hecho... » (ex. 8);

- les raisons qui ont poussé la direction du musée à exposer la statue: "The patrons of the exhibition insist... » (ex. 1), « The model had sparked controversy... » 
(ex. 4), « organizatorji so se branili z argumentom... » (ex. 6), « Los responsables del museo se han defendido... » (ex. 8);

- les polémiques avant l'exposition de la statue: "L'arrivo della statua [...] ha suscitato forti polemiche » (ex. 3), « The model had sparked controversy » (ex. 4), «posjetitelji su nakon višemjesečnih kontroverzi » (ex. 5), «Vključitev lutke [...] je vzbudila številne kritike in negodovanja... » (ex. 6), « ...hatte es eine Kontroverse über die Frage gegeben, ob der Diktator überhaupt präsentiert werden dürfte » (ex. 7), « La presencia de la figura [...] había generado duras críticas » (ex. 8).

- la description de l'homme: « cet ancien policier, âgé de 41 ans et aujourd'hui au chômage » (ex. 2), " in Berlin wohnenden Mann » (ex. 7), " el responsable de la decapitación vive en el barrio de Kreuzberg... » (ex. 8);

- la description de la statue et de sa position: « La statua [...] ritraeva Hitler negli ultimi giorni... » (ex. 3), "The model was perched behind a desk... [...] It portrayed Hitler in defeat... » (ex. 4), " Hitler je prikazan kao uništen starac na kraju života » (ex. 5), « Lutka [...] kaže Hitlerja, ko sedi za mizo... » (ex. 6), « die Figur sitzt in einem Diorama... » (ex. 7), « la figura lo muestra ya en su época de decadencia... » (ex. 8); - certaines informations sur le musée: «Il nuovo museo [...] è la terza succursale... » (ex. 3), " Das neue Berlin Wachsfigurenkabinett ist nach London und Amsterdam... » (ex. 7), « la filial del Museo Tussaud en Berlín es la tercera en Europa,... » (ex. 8).

- des informations sur les autres statues exposées dans le musée: « Uz Hitlera [...] nova podružnica galerije voštanih figura prikazuje još sedamdesetak osoba... » (ex. 5).

Dans les deux premiers cas, il s'agit de séquences explicatives (qui répondent à la question: " Pourquoi l'homme a-t-il agi ainsi? » et " Pourquoi la direction a-t-elle exposé la statue? »). Dans les autres cas, il s'agit pour la plupart de séquences de type descriptif.

\section{CONCLUSION}

Partant de l'hypothèse qu'un fait divers, portant sur le même thème, a une structure comparable dans différentes langues et qu'il contient au moins une séquence narrative, nous avons analysé huit faits divers en français, espagnol, italien, allemand, anglais, slovène et croate; deux articles courts et six articles plus longs, donc deux articles qui se présentent sous forme condensée et six articles, présentés sous forme expansée (Petitjean $1987: 83$ ).

Tous les articles analysés contiennent une partie narrative qui apparaît au début du texte. Dans le premier exemple, c'est la narration qui prédomine; il n'y a qu'une seule proposition qui n'entre pas dans le contexte de la narration. Le deuxième exemple est composé de deux séquences, l'une narrative et l'autre explicative. Pourtant, l'intention de l'auteur de l'article est, à notre avis, surtout de raconter l'histoire; pour cela, la dominante textuelle est narrative.

Dans les textes plus longs, la narration se trouve toujours au début du texte et le texte continue par des séquences d'autres types, surtout des explications et des descriptions. Il n'y a qu'un seul texte qui contienne deux séquences narratives (exemple 8). 
L'hypothèse du départ est confirmée: la structure de tous les faits divers analysés se ressemble et ils contiennent tous au moins une séquence narrative.

Les thèmes traités dans les textes plus longs se répètent. On retrouve en effet les thèmes suivants: les raisons du comportement de l'interpellé (six exemples), les raisons qui ont poussé la direction du musée à prendre la décision d'exposer la statue (4 exemples), l'évocation des polémiques avant l'exposition (6 exemples), la description de l'homme (3 exemples), la description de la statue (6 exemples), une information sur le musée (4 exemples). On pourrait qualifier les séquences traitant ces thèmes de descriptives et d'explicatives. Les faits divers analysés sont donc vraiment hétérogènes (Petitjean 1987 : 73), mais il y a toujours une séquence narrative au début du texte.

Dans ce bref passage en revue des faits divers à travers les langues et les cultures, nous nous sommes concentrés sur l'aspect textuel des textes. Il serait entre autres intéressant d'analyser les faits divers du point de vue énonciatif, que nous n'avons pas approfondi dans notre étude. Il serait tout aussi utile de refaire l'analyse avec un corpus plus large.

\section{Bibliographie}

ADAM, Jean-Michel (1997) Les textes - types et prototypes. Paris: Nathan/HER.

ADAM, Jean-Michel (2001) « Genres de la presse écrite et analyse de discours. » Semen 13,

Genres de la presse écrite et analyse de discours [en ligne], mis en ligne le 30 avril 2007,

URL: . revues.org/document2597.html. Consulté le 30 juin 2008.

ADAM, Jean-Michel (21999 [1994]) Le texte narratif. Paris: Nathan Université.

BÉGorre Bret, Cyrille/Raphaël Giraud/Sébastien Miller (2004) « Le fait divers et la nouvelle rhétorique démocratique. » Le Banquet 19, 2004/1. Consulté le 30 juillet 2008.

Charaudeau, Patrick, Maingueneau, Dominique (éditeurs) (2002) Dictionnaire d'analyse du discours. Paris: Seuil.

Eco, Umberto (1985) Lector in fabula. Paris: Grasset.

LITS, Marc (2001) « Le fait divers: un genre strictement francophone? » Semen 13, Genres de la presse écrite et analyse de discours [en ligne], mis en ligne le 30 avril 2007, URL: . revues.org/document2628.html. Consulté le 2 juillet 2008.

Petitjean, André (1978) «Les faits divers: polyphonie énonciative et hétérogénéité textuelle. » Langue française 74, La typologie des discours, 73-96.

TISSET, Caroline (2000) Analyse linguistique de la narration. Paris: SEDES.

DuBIED, Annik (2001) «Invasion peritextuelle et contamination médiatiques. Le 'fait divers', une catégorie complexe ancrée dans le champ journalistique. » Semen 13, Genres de la presse écrite et analyse de discours [en ligne], mis en ligne le 30 avril 2007, URL: . revues.org/document2633.html. Consulté le 5 juillet 2008. 
López Alonso, Covadonga/Arlette Séré de Olmos (1996) « Typologie des textes et stratégies de la compréhension en langue étrangère "Etudes de linguistique appliquée 104 (Comprendre les langues voisines), 441-451.

TAgliante, Christine (1994) La classe de langue. Paris: CLE international.

Articles analysés:

Exemple 1: http://www.shortnews.com/start.cfm?id=71829

Exemple 2:

http://www.lefigaro.fr/flash-actu/2008/07/07/01011-20080707FILWWW00368-berlin-la-stat ue-d-hitler-sera-reparee.php

Exemple 3:

http://www.euronews.net/it/article/05/07/2008/hitler-headless-after-man-attacks-waxwork-inberlin/

Exemple 4: http://www.newser.com/story/31706/wax-hitler-loses-its-head.html

Exemple 5: http://www.nacional.hr/articles/view/47055/

Exemple 6: http://www.delo.si/clanek/63244

Exemple 7:

http://www.nzz.ch/nachrichten/panorama/museumsbesucher_reisst_hitler_kopf_ab_wachsf igurenkabinett_berlin_1.777102.html

Exemple 8:

http://www.elpais.com/articulo/gente/Hitler/decapitado/elpepugen/20080705elpepuage_1/Tes 


\section{Povzetek \\ ČLANEK IZ (ČRNE) KRONIKE: PRIPOVED Z NAPAKO?}

Avtorica se v prispevku ukvarja s članki, ki jih v časopisih najdemo v rubriki "(črna) kronika". Članki iz (črne) kronike so, kot ugotavljajo nekateri od citiranih avtorjev, sorazmerno slabo raziskani. Lits (2001: 2) navaja svoje izkušnje s tujimi študenti in se sprašuje, ali ne gre za izključno frankofonski žanr besedila. Kar zadeva tip besedila, so članki na prvi pogled pripovednega tipa, pogosto pa se po podrobnejši analizi pokaže, da se v njih pojavljajo sekvence različnih tipov. Avtorica analizira osem člankov iz črne kronike na isto temo, v naslednjih jezikih: angleškem, nemškem, francoskem, španskem, italijanskem, slovenskem in hrvaškem. Pri tem izhaja iz predpostavke, da bo struktura besedila podobna v vseh jezikih in da bodo vsi članiki vsebovali vsaj eno pripovedno sekvenco. Pri krajših člankih (primer 1 in 2) pripovedna sekvenca zavzema večino besedila, pri daljših člankih (vsi ostali primeri, razen primera 8 , ki vsebuje dve sekvenci pripovednega tipa) pripovedna sekvenca služi kot uvod. Obravnavane teme so podobne v vseh člankih, poleg pripovednih pa v člankih najdemo še sekvence opisnega in razlagalnega tipa; hipoteza je torej potrjena. 